\title{
Parameter extraction from experimental PEFC data using an evolutionary optimization algorithm
}

\author{
M. Zaglio ${ }^{1, a}$, G. Schuler ${ }^{1}$, A. Wokaun ${ }^{1}$, J. Mantzaras ${ }^{2}$, and F.N. Büchi ${ }^{1}$ \\ 1 Electrochemistry Laboratory, Paul Scherrer Institut, 5232 Villigen PSI, Switzerland \\ 2 Combustion Research Laboratory, Paul Scherrer Institut, 5232 Villigen PSI, Switzerland
}

Received: 10 May 2010 / Received in final form: 8 December 2010 / Accepted: 4 March 2011 Published online: 20 May 2011 - (c) EDP Sciences

\begin{abstract}
The accurate characterization of the parameters related to the charge and water transport in the ionomer membrane of polymer electrolyte fuel cells (PEFC) is highly important for the understanding and interpretation of the overall cell behavior. Despite the big efforts to experimentally determine these parameters, a large scatter of data is reported in the literature, due to the inherent experimental difficulties. Likewise, the porosity and tortuosity of the gas diffusion layers affect the membrane water content and the local cell performance, but the published data are usually measured ex-situ, not accounting for the effect of clamping pressure. Using a quasi two-dimensional model and experimental current density data from a linear cell of technical size, a multiparameter optimization procedure based on an evolutionary algorithm has been applied to determine eight material properties highly influencing the cell performance. The optimization procedure converges towards a well defined solution and the resulting parameter values are compared to those available in the literature. The quality of the set of parameters extracted by the optimization procedure is assessed by a sensitivity analysis.
\end{abstract}

\section{Introduction}

Fuel cells are complex, three-dimensional electrochemical reactors, wherein the electrochemical processes are intricately coupled with multiphase, multispecies mass transport and with energy and charge conduction through very different phases. The complexity of the system requires also model based approaches for in-depth comprehension, hence modeling in the field of polymer electrolyte fuel cells (PEFC) has become a complementary requirement to experiments [1]. One core level of modeling is the description at the cell level, which is of high importance for the understanding of important phenomena such as water management, species transport at all scales, current distribution, and cell local and integral performance.

On the cell level, quasi two-dimensional models [2-4] can describe the spatial variations along the cell channels as well as the main gradients through the different domains of a PEFC perpendicular to the channels, thus extending the capability of one-dimensional models to complete cells with significant reduction in calculation efforts as compared to three-dimensional approaches. Quasi twodimensional models are for these reasons particularly suitable to run optimization procedures that require a large number of simulations.

\footnotetext{
a e-mail: maurizio.zaglio@psi.ch
}

Local and integral fuel cell performances are strongly determined by mass and charge transport properties of the membrane and GDL (gas diffusion layer). However, the intrinsic difficulties in measuring the membrane charge and mass transport properties have led to a large scatter of data reported in the literature, even for ex-situ experiments [5-14]. The use of various experimental techniques and of different operating conditions possibly explains the inconsistencies, but also handling and pretreatment of the membrane are important in determining its water content [12], upon which all the considered parameters are critically dependent. Furthermore, no direct experimental characterization of these parameters in operating cells has been conducted, and the employed ex-situ setups are generally simplifying the real conditions in an operating fuel cell. Likewise, porosity and tortuosity measurements of the GDL are mainly conducted ex-situ without taking into account the clamping pressure in a real cell $[15,16]$, which can crucially affect these parameters [17].

Optimization algorithms provide the possibility to extract material properties, which are difficult to measure in-situ. Still, parameter fitting procedures for PEFCs are scarce in the literature. Mo et al. [18] apply a method based on genetic algorithms to fit coefficients to experimental data. More frequently, optimization procedures have been applied in the design of cell components. Peng et al. [19] use adaptive simulated annealing for designing the hydrogen distribution channels, so as to maximize 
the reaction efficiency. Using the sequential quadratic programming method $\mathrm{Na}$ and Gou [20] maximize the fuel efficiency in order to minimize the costs of the cell, including stack and balance of plant. Bao et al. [21] optimize the air stoichiometry ratio and the cathode outlet pressure using a genetic algorithm. A code also based on a genetic algorithm is used by Mohamed and Jenkins [22] to optimize the stack configuration, in terms of number of cells and cell area. Zaglio et al. [23] compare different optimization procedures applied to the extraction of fuel cell parameters, one based on Newton's method and one based on an evolutionary algorithm (EA [24]). They both converged to a very similar solution, but Newton's method is not able to avoid eventual local minima in the solution field, and the solution is not ensured to be the global best. Furthermore, in a discontinuous solution field, the solution could depend on the initial seeking point. The EA instead has the capability to converge to the global best solution, given an adequate number of individuals and generations, and it does not require an initial seeking point, but relies on a stochastic initialization step.

An optimization procedure based on an evolutionary algorithm is pursued in the present study to determine a set of eight material properties related to the membrane and to the gas diffusion layers, minimizing the difference between experimental and simulated local current distribution along the channels, simultaneously for different operating conditions. EAs are effectively used in minimization/maximization problems, and are able to converge towards the global best solution even in a non-linear and discontinuous solution field [25].

A quasi two-dimensional model of a linear cell previously developed by Freunberger et al. [2] is used in the fitting procedure. The model is able to describe the influence of different operating conditions on the species and field distribution in the along-the-channel and throughthe-MEA (membrane electrode assembly) directions. The current density distribution data used is collected at four different conditions, in coflow and counterflow configurations, and at different loads, to have a broad range of membrane humidification conditions for fitting the investigated parameters.

\section{Experimental data}

Current density distributions used in the fitting procedure are measured in a linear PEFC of technical size as described in $[2,26]$. The flow field comprises 28 straight and parallel $400 \mathrm{~mm}$ long channels that cover an active area of $200 \mathrm{~cm}^{2}$. As electrochemical components, gas diffusion electrodes E-TEK ELAT V2.1 with a platinum loading of $0.5 \mathrm{mg} \mathrm{cm}^{-2}$ and Nafion 112 are used. The local current is measured in 10 segments equally distributed between cell inlet and outlet, using a current mapping technique based on a semisegmented cathode endplate and an unsegmented MEA as described in [27,28]. Hydrogen and air are used as reactants, with flow direction set to either coflow or counterflow. The operating parameters are described in Table 1 and the experimental data is shown in Figure 1.
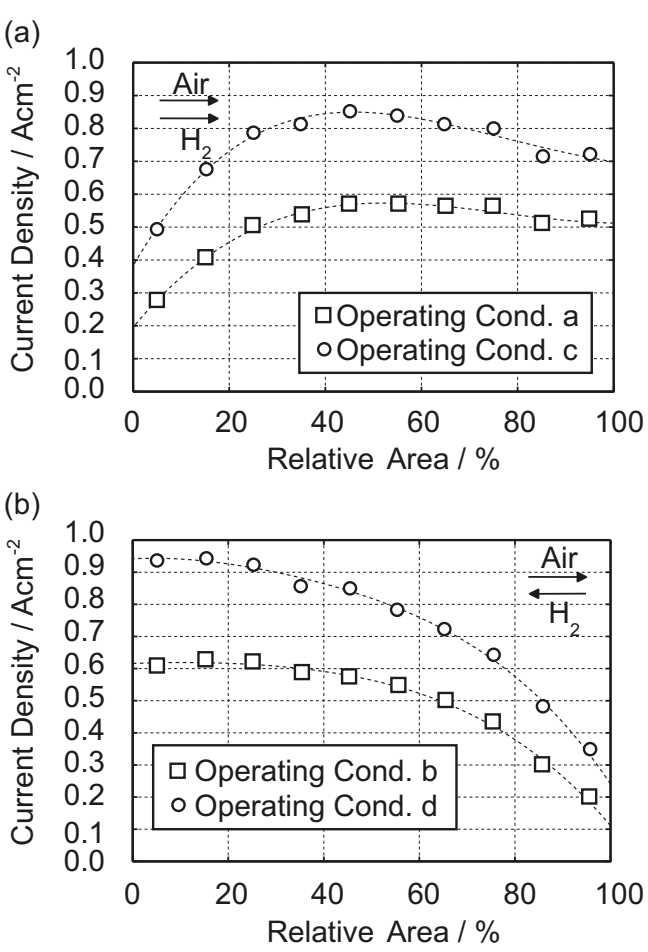

Fig. 1. Measured current density distributions used in the fitting procedure: (a) coflow and (b) counterflow. Other operating parameters are listed in Table 1 . The relative area is given by the cell width and the relative length of the linear channels, calculated from the air inlet. Lines for guiding the eye.

Table 1. Experimental operating conditions a to c: CO coflow, CT counterflow.

\begin{tabular}{|c|c|c|c|c|}
\hline Parameter & $a$ & $b$ & $c$ & $d$ \\
\hline Current density $\left[\mathrm{A} \mathrm{cm}^{-2}\right]$ & \multicolumn{2}{|c|}{0.5} & \multicolumn{2}{|c|}{0.75} \\
\hline Anode inlet relative humidity & \multicolumn{4}{|c|}{0.0} \\
\hline Cathode inlet relative humidity & \multicolumn{4}{|c|}{1.0} \\
\hline Flow direction & $\mathrm{CO}$ & $\mathrm{CT}$ & $\mathrm{CO}$ & $\mathrm{CT}$ \\
\hline Hydrogen stoichiometry & \multicolumn{4}{|c|}{2.0} \\
\hline Air stoichiometry & \multicolumn{4}{|c|}{2.0} \\
\hline Cell temperature $\left[{ }^{\circ} \mathrm{C}\right]$ & \multicolumn{4}{|c|}{70} \\
\hline Gas pressure [bar] & \multicolumn{4}{|c|}{1.5} \\
\hline
\end{tabular}

The fitting procedure is performed on a Intel Core2 CPU 6600@2.40 GHz work station with 1.0 GB RAM.

\section{Optimization procedure}

Optimization procedures are used to minimize/maximize an objective function depending on the parameters under fitting. Here the objective function is the difference between experimental and simulated current density distribution in the along-the-channel direction for different operating conditions. While seeking the global minimum of this function, the procedure converges towards the best set of parameters under fitting, thus defining the corresponding parameters in the real cell. The fitting algorithm requires (i) experimental data at well defined operating 
conditions, (ii) a model able to simulate the behavior of the experimental setup, (iii) the parameters to fit and finally, (iv) the accepted range for each parameter under fitting, to define the seeking field.

\subsection{Evolutionary algorithm}

In EAs the evolution principle is applied in the search of the global best solution to an optimization problem [25]. In the last 40 years many different variants of evolutionary algorithms have been developed, all of them sharing the same concept: given a population of individuals (possible solutions) the environmental pressure determines a natural selection, only the fittest survives, which increases the fitness of the population. An objective function is used as the fitness measure. Based on this fitness, recombination and mutation are applied to some of the best candidates and the process is iterated until an offspring candidate satisfies the termination condition. The evolutionary algorithm used here is described in detail in [24] and is based on the code developed at the "Aerothermochemistry and Combustion Systems Laboratory", ETH Zürich (Switzerland). The initialization step is performed on a randomly generated population. The objective function to be minimized is $Y$ in equation (1), the root mean square (RMS) deviation between the experimental and simulated data:

$$
Y=\sqrt{\sum_{i=1}^{n_{d}}\left(j_{i}^{\exp }-j_{i}^{\operatorname{sim}}\right)^{2}}
$$

where $j_{i}$ is the local current density at the segment $i$ along the channel and $n_{d}$ the number of nodes along the channel.

The procedure used in this work is schematically shown in Figure 2. The parent selection step is responsible for raising the quality of the population. Specifically, high quality individuals have a high chance to become parents. However, low quality individuals also get a smaller chance, such that the search procedure does not lock to a local optimum [25].

EA are well adapted for the problem because the optimization level (solid lines boxes in Fig. 2) and the simulation level (dashed line box in Fig. 2) are well distinguished. An existing model can then be used and it is treated as a black box by the EA. Only an interface is needed where the coefficients under optimization are passed to the simulation level, where the corresponding objective function is computed and transferred back to the optimization level.

\section{$3.21+1-\mathrm{D}$ model}

The seeking procedure for the global minimum in the solution field requires a large number of simulations. For this reason, it is important to keep the computational cost for a single run reasonably low. This is the case for the quasitwo dimensional model [2] used in the fitting procedure, which has previously been developed for linear PEFCs of technical size. Such cells are characterized by a high aspect

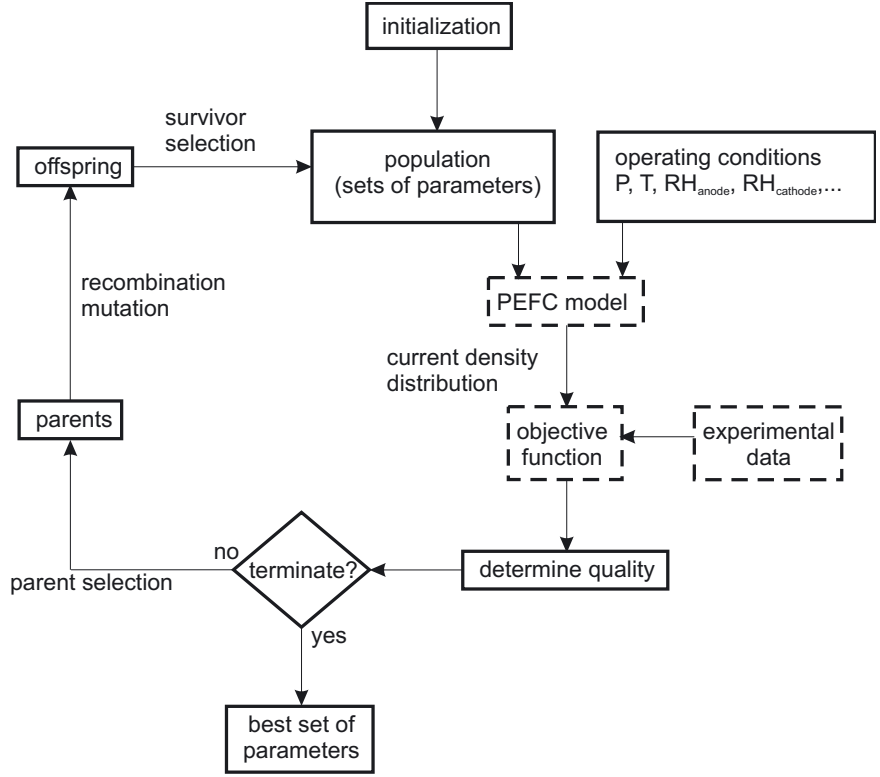

Fig. 2. General scheme of the EA fitting procedure.

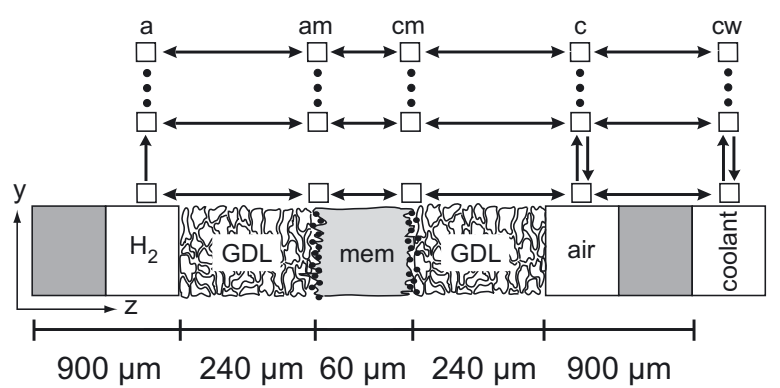

Fig. 3. PEFC model domain. $y$ : along the channel direction, $z$ : through he MEA direction. a: anode channel, am: anode GDL-membrane interface, cm: cathode GDL-membrane interface, c: cathode channel, cw: coolant water channel. The transport pathways are denoted by the arrows. Dots represent repeating units in the through-the-MEA direction (from [26]).

ratio, tens of $\mathrm{cm}$ long channels and several hundreds of $\mu \mathrm{m}$ of MEA thickness. Transport in the along-the-channel direction is then by far dominated by convection in the channels, while transport in the MEA can be neglected in this direction. Even though it has been experimentally shown that the local current density in the direction normal to the channels is also inhomogeneous [29-31], it seems reasonable for a multichannel configuration to neglect such gradients in parameters distributions. This assumption is further justified by the decreased computational cost of the quasi-two dimensional model compared to a three dimensional approach. The model has been discussed in detail in [2] and is therefore only summarized here. It incorporates all important processes in the direction normal to the MEA ( $z$ in Fig. 3): multicomponent mixture, multiphase transport of gaseous species, transport of water in the membrane by diffusion and electro-osmotic drag, heat generation and transport in the cross section of the cell, electrochemical reaction at the interface. Transport along 
the channels $(y)$ is described by plug-flow without axial dispersion. Boundary conditions governing the local transport through the MEA vary along the flow direction with the species composition and the temperature in the channels. The local reactant consumption, water balance, and heat source in turn serve as source terms for the media flow in the channels. The resulting quasi two-dimensional model is represented by slices through the cell coupled by the flow in the channels only, as shown in Figure 3.

The current density distribution to be compared with the experiments is calculated under the assumption of isopotential flow field plates. Because the composition of local overpotential varies along the channel, the local current density is adjusted to fulfill the condition:

$$
U=E_{0}-\eta_{\text {act }}-\eta_{\text {conc }}-\eta_{R}
$$

with $E_{0}$ the equilibrium potential, $\eta_{\text {act }}, \eta_{\text {conc }}, \eta_{R}$ the activation, concentration and membrane ohmic overpotentials, respectively. At each iteration, according to the local species, temperature and current density distribution, the local overpotentials are calculated. The local current density is subsequently adjusted using equation (2) with the uniform average voltage. Further, the current profile is leveled to meet the specified total current. When both conditions are fulfilled, the new current density distribution is used at the next iteration to calculate the new sink/source terms and the new properties distribution in the different domains.

\subsection{Determination of the reaction kinetic parameters}

As stated in the previous section, to determine the local current density, three losses are taken into account: activation, ohmic, and concentration overpotentials. Ohmic and concentration polarization are determined by the charge and mass transport in the different domains of the model, and therefore by those fitted parameters presented in the following sections. The activation overpotential is a measure of the activity of the electrodes. It pertains to the specific electrochemical reaction, and it is mainly a function of the electrode characteristics: type and quantity of catalyst, dimension and distribution of the catalyst particles, active surface. Among the two activation overpotentials, the anode activation is negligible compared to the cathode activation overpotential $[32,33]$. Hence, the cathodic overpotential will be simply referred to as the activation overpotential, neglecting the small anodic polarization.

In the model, the voltage drop due to activation overpotential is calculated by the Tafel equation, equation (3):

$$
\eta_{\text {act }}=\left(\frac{R T}{\alpha n F}\right) \ln \left(\frac{j}{j^{o}}\right) .
$$

The exchange current density $j^{\circ}$ is expressed as function of the temperature at the reactive interface:

$$
j^{o}=j^{\text {ref }} \exp \left[8895\left(\frac{1}{303}-\frac{1}{T}\right)\right] .
$$

Table 2. Kinetic parameters used in the model.

\begin{tabular}{lc}
\hline Parameter & Value \\
\hline$\alpha$ & 0.69 \\
$j^{\text {ref }},\left[\mathrm{A} \mathrm{m}^{-2}\right]$ & 0.0052 \\
\hline
\end{tabular}

As shown in equations (3) and (4), the transfer coefficient $\alpha$, and the reference exchange current density $j^{\text {ref }}$, are the two material parameters determining the activation overpotential. Due to the predominance of this overpotential over the other losses in the low current density range, these parameters are fitted to minimize the difference between the calculated and the experimental polarization curve, in 14 points at different pressures, temperatures and loads. Four points are measured at $0.04 \mathrm{~A} \mathrm{~cm}^{-2}$, varying the cell outlet pressure from 1 bar to $1.2,1.5$ and 2 bar respectively, keeping the cell temperature at $70{ }^{\circ} \mathrm{C}$, the air and fuel stoichiometry at 1.3 and the inlet relative humidities at 1.0. Four further points are collected for the same conditions but at $0.12 \mathrm{~A} \mathrm{~cm}^{-2}$. Finally, six points are measured varying the cell temperature from $60{ }^{\circ} \mathrm{C}$ to $70{ }^{\circ} \mathrm{C}$ and $75^{\circ} \mathrm{C}$ respectively, at a constant cell outlet pressure of $1.5 \mathrm{bar}$, three points at $0.04 \mathrm{~A} \mathrm{~cm}^{-2}$ and the other three at $0.12 \mathrm{~A} \mathrm{~cm}^{-2}$. Feeding gas stoichiometries and relative humidities are not changed, and due to the negligible difference in this range between coflow and counterflow polarization curves, only data in coflow configuration have been considered. Having only two parameters under fitting, a brute force method has been used: a seeking field has been set for each parameter, and the whole seeking field has been scanned to determine the best set of the two parameters. Results are shown in Table 2.

\subsection{Fitting parameters}

For a given set of electrochemical components and operating conditions, water and charge transport in the membrane and the transport properties of the GDL are the most influential parameters determining the current distribution.

Four fitted parameters are therefore related to the material properties of the porous domains, namely porosity and tortuosity at the anode and cathode sides. These domains are treated as homogeneous in the model. In this respect, the calculated values are averages of the local parameters over the entire domain. Four additional material properties being fitted are those affecting transport of water and protons in the membrane, namely water diffusion coefficient, electro-osmotic drag coefficient, ionic conductivity and water sorption isotherm, which are highly dependent on the membrane local water content and local temperature. A characteristic curve for each property is therefore provided to the model, to simulate the dependency on the local membrane conditions. To preclude convergence of the algorithm towards unrealistic values, maximum and minimum boundaries are set for each property taking into account literature ex-situ experimental data. 


\subsubsection{Experimental data in the literature}

Ohmic resistance in the membrane is strictly dependent on local water content. Membrane local humidification results from the water sorption at the interface with the catalyst layers and then from its transport by the electro-osmotic drag and diffusion, both depending on the water content and often acting in opposing directions [34].

Proton and water transport in Nafion are well documented properties. Extensive efforts have been made to investigate water diffusion coefficient, electro-osmotic drag, ionic conductivity, and membrane water uptake, with very different experimental procedures. However, the values proposed in the literature show a large scatter, and are generally measured ex-situ due to the difficulty of performing measurements in an operating cell, eventually simplifying the real conditions the membrane is exposed to.

The published data reviewed herein are related to Nafion membranes with an equivalent weight of 1100 . Yeo and Eisenberg [35] investigated as early as 1977 the temperature dependency of diffusion coefficients based on gravimetric water uptake measurements. Their measurements are still often used to obtain data at high temperatures from experiments performed at room temperature, [6,36]. More recently, IR spectroscopy [37], Nuclear Magnetic Resonance (NMR) [7,8,38], simple fitting procedures based on measured water content leaving the cell [36], as well as flow permeation and sorption methods $[13,39]$ have been used to determine the water diffusion coefficient in membranes. Different dependencies of the diffusion coefficient on the membrane water content, varying from linear (Fuller [38]), Nguyen and Vanderborgh [8]) to highly non-linear (Zawodzinski et al. [7], van Bussel et al. [40], Rivin et al. [39]) have been reported, as shown in Figure 4a.

The electro-osmotic drag coefficient has been measured with special drag cells [12], the activity gradient method [41,42], and electrophoretic nuclear magnetic resonance (NMR) [43]. Most of the measurements report a constant coefficient over a wide range of membrane humidity, which drops to zero for dry membranes, [12,41,42], with values ranging from 1 to 3 at equilibrium with liquid water, see Figure 4b.

Values for the ionic conductivity of Nafion also show a scatter. The conductivity has been investigated predominantly using $\mathrm{AC}$ impedance $[7,10,44]$. A variety of environments has been employed including water [7], water vapor [10,12,44-46] and humidified gases [47] at temperatures between 20 and $95{ }^{\circ} \mathrm{C}$. Verbrugge et al. [11] and Marechal et al. [48] showed that Nafion conductivity can more than double between 20 and $80^{\circ} \mathrm{C}$, while most of the other measurements were taken at $25{ }^{\circ} \mathrm{C}$, far from the standard operating conditions of a PEFC $\left(60-80^{\circ} \mathrm{C}\right)$, see Figure 4c.

The amount of water in the membrane is critical, as all the other parameters in this section directly depend upon it. Many investigators have studied the uptake of water in membranes, based on spectroscopic, gravimetric and other methods $[12,13,49,50]$. Generally, the water uptake isotherm has a typical trend: it has a sharp initial slope,
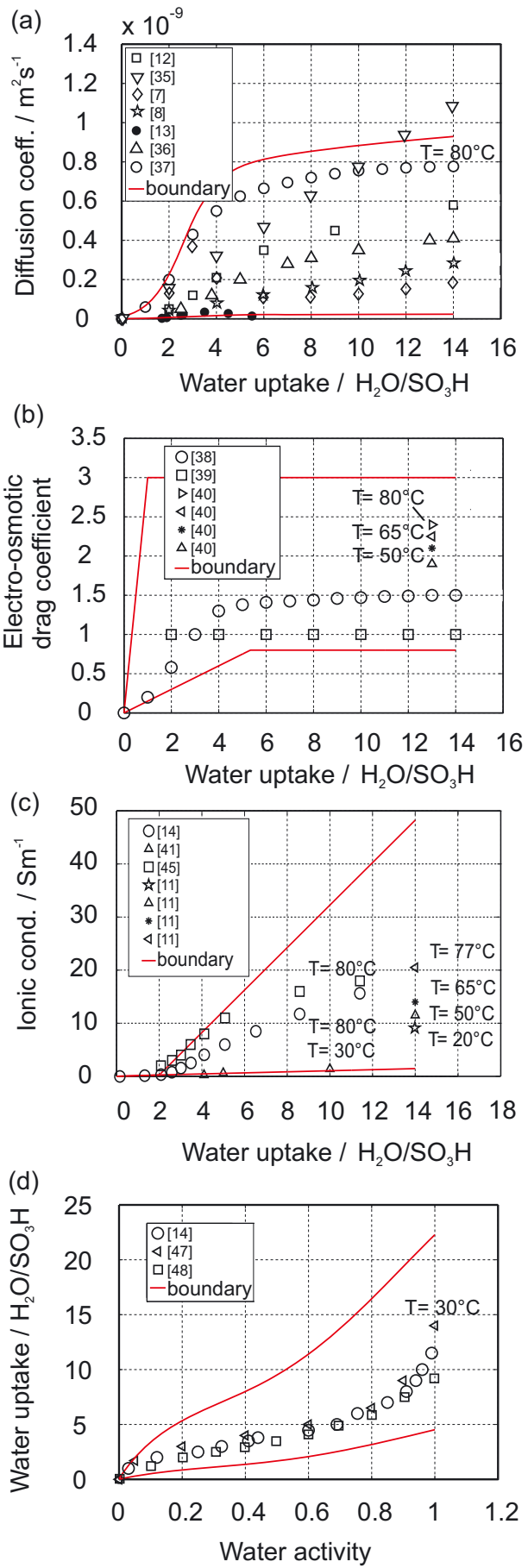

Fig. 4. (Color online) Published membrane properties under fitting: (a) water diffusion coefficient, $T=30{ }^{\circ} \mathrm{C}$ unless otherwise indicated, (b) electro-osmotic drag coefficient, $T=25{ }^{\circ} \mathrm{C}$ unless otherwise indicated, (c) ionic conductivity and (d) water uptake isotherm, $T=80^{\circ} \mathrm{C}$ unless otherwise indicated.

leveling off at a water activity of about 0.2 , followed by an extended region of a gradually increasing slope that rises again sharply above a water activity of 0.7 . A certain discrepancy is reported between the maximum value that can be taken up by the membrane at high water activity, ranging from $9.8 \mathrm{H}_{2} \mathrm{O} / \mathrm{SO}_{3} \mathrm{H}[49]$ to $14.0 \mathrm{H}_{2} \mathrm{O} / \mathrm{SO}_{3} \mathrm{H}$ [50]. 
All the published values fall in this range, [7,14,51], see Figure 4d.

Water transport in the porous layers affects the water transport in membrane and depends on the characteristics of the porous media used. GDLs are typically multilayered carbon fiber based porous materials containing a macro-porous substrate or backing layer which enhances the electrical contact and influences the water transport. Mercury porosimetry $[15,52]$ and the immersion method [53] are used to determine the bulk porosity of GDLs. Tortuosity is usually determined from the porosity and the effective diffusivity measurements [16,54]. However, the effective tortuosity in a complete cell is different from the through-plane measured one in a porous layer, due to the longer path under the ribs of the bipolar plate. These properties are highly affected by the clamping pressure applied to the cell [17].

\subsubsection{Parameters expressions}

Despite the large scatter of data, common properties of the four membrane parameters can be found. For this reason, characteristic curves are provided to the model to simulate their dependency on the local membrane conditions. Each curve is composed of one or more coefficients that determine its shape.

The sigmoidal shape in equation (5) is based on the equation presented in [55] describing the water diffusion in the membrane as a function of local water content and temperature:

$$
\begin{aligned}
D_{w}= & A \times 10^{-11}\left[1+\tanh \left(\frac{\lambda-2.5}{1.4}\right)\right]\left(\frac{\lambda}{25}\right)^{0.15} \\
& \times \exp \left(2416\left(\frac{1}{303}-\frac{1}{T}\right)\right)
\end{aligned}
$$

where $A$ is a scaling parameter being fitted and $\lambda$ is the membrane water content.

According to the reviewed data, the water drag coefficient, $\chi$, is constant over a wide range of membrane water content. It is therefore described here by $B$ in equation (6) setting the slope of the curve starting from zero water content, and $C$ defining the maximum value up to $\lambda=14$ :

$$
\chi=\left\{\begin{array}{cl}
0.3 B \lambda & \text { if } \chi<C \text { and } \lambda \leq 14 \\
C & \text { if } \chi \geq C \text { and } \lambda \leq 14
\end{array}\right.
$$

Based in Figure 4c, the ionic conductivity is very low at $\lambda \leq 2$, and increases linearly at higher water content:

$$
K= \begin{cases}0.125 \lambda & \text { if } \lambda \leq 2 \\ D \lambda-2 D+0.25 & \text { if } \lambda>2\end{cases}
$$

where $D$ is the coefficient under fitting.

The water sorption isotherm is described by a more complicated polynomial equation, with two coefficients to fit, $E$ and $F$ in equation (8):

$$
\begin{aligned}
\lambda= & \left(1+\frac{E}{a_{w}+F}\right)\left(2.0 a_{w}^{5}-5.2 a_{w}^{4}+11.0 a_{w}^{3}\right. \\
& \left.-8.8 a_{w}^{2}+3.9 a_{w}+0.0063\right)
\end{aligned}
$$

Table 3. Seeking field for each coefficient and material property under fitting.

\begin{tabular}{lcccc}
\hline Property & Coeff. & Min & Max & Discr. interval \\
\hline Diffusion & $A$ & 0.5 & 20 & 0.005 \\
El-osmotic drag & $B$ & 0.5 & 10 & 0.01 \\
El-osmotic drag & $C$ & 0.8 & 3.0 & 0.005 \\
Conductivity & $D$ & 0.1 & 2.5 & 0.01 \\
Sorption & $E$ & 5.0 & 20 & 0.005 \\
Sorption & $F$ & 0.2 & 8 & 0.005 \\
Porosity, anode $\epsilon_{\text {an }}$ & & 0.3 & 0.8 & 0.01 \\
Tortuosity, anode $\tau_{\text {an }}$ & & 2 & 10 & 0.01 \\
Porosity, cathode $\epsilon_{\text {cat }}$ & & 0.3 & 0.8 & 0.01 \\
Tortuosity, cathode $\tau_{\text {cat }}$ & & 2 & 10 & 0.01 \\
\hline
\end{tabular}

where $a_{w}$ is the water activity at the membrane/porous layer interfaces:

$$
a_{w}=\frac{P_{\mathrm{vap}}}{P_{\mathrm{sat}}(T)} .
$$

Therefore, six coefficients related to the transport phenomena in the membrane are fitted. The limit values are set based on the published measurements as given in Table 3 and Figure 4. Additionally, porosity and tortuosity of the anode and cathode GDLs are included in the fitting procedure. Their limits specify a large seeking field, due to a lack of precise information in the literature.

\section{Results and discussion}

The ten coefficients used to describe the eight material properties related to the membrane and to the porous GDLs have been fitted with the procedure described in Section 3. The experimental data used for minimizing the objective function are current density distributions at different loads and different flow configurations, for a dry anode and well humidified cathode feed, see Figure 1. The local membrane humidity is therefore very different and allows the fitting of each membrane parameter over a wide range of membrane water content.

The termination condition in the EA is set by the number of generations, fixed to 15 because this number is adequate to reach the minimum value of the objective function. At the stochastic initialization step, the population is composed of 25 different sets of parameters (individuals), while at each generation the population is composed of 30 different individuals. Each individual is provided to the model for evaluation of the objective function (Eq. (1)). In Figure 5 the maximum and the minimum values assumed by the objective function are shown for each generation. The main features of this optimization algorithm are recognized in this plot. After the initialization step, the population at the first generation is composed of individuals with very different qualities. At each generation, by the selection of the best individuals as the parents of the next generation, the fitting procedure is able to restrain the seeking field closer to the neighborhood of the best solution. To avoid the convergence to a local minimum, the 
M. Zaglio et al.: PEFC parameter extraction using an evolutionary optimization algorithm

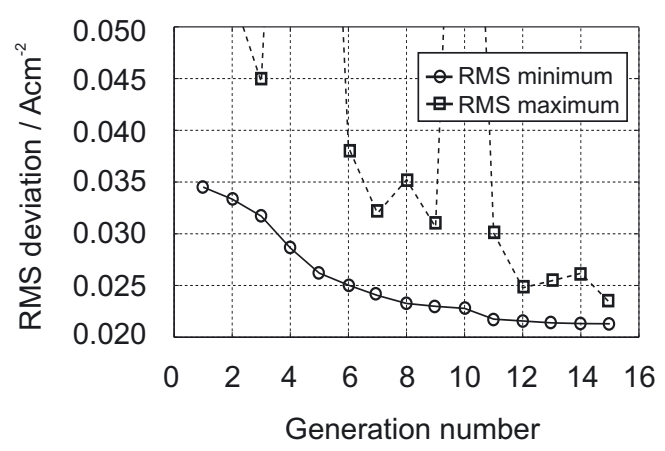

Fig. 5. RMS maxima and minima for fitting with evolutionary algorithm at each generation.

Table 4. Best set of coefficients and material properties resulting form the fitting procedure.

\begin{tabular}{lcc}
\hline Property & Coeff. & Value \\
\hline Diffusion & $A$ & 0.9900 \\
El-osmotic drag & $B$ & 4.47 \\
El-osmotic drag & $C$ & 1.065 \\
Conductivity & $D$ & 1.37 \\
Sorption & $E$ & 10.99 \\
Sorption & $F$ & 3.585 \\
Porosity anode $\epsilon_{\text {an }}$ & & 0.55 \\
Tortuosity anode $\tau_{\text {an }}$ & & 8.00 \\
Porosity cathode $\epsilon_{\text {cat }}$ & & 0.36 \\
Tortuosity cathode $\tau_{\text {cat }}$ & & 7.34 \\
\hline
\end{tabular}

algorithm uses mutation and recombination to generate individuals that are resulting from the best solutions at the previous generation, but are exploring different areas of the solution field. These individuals may have a lower quality compared to the others, as shown in Figure 5 at generation 4, 5, 8, 10 and 14. The complete EA fitting procedure takes about $36 \mathrm{~h}$. The computational cost of the current density distribution calculation in one single condition is highly dependent on the parameters under investigation and the flux configuration (co-/counterflow), and varies between $30 \mathrm{~s}$ and 5 min.

The parameters resulting form the fitting procedure are listed in Table 4 and those related to the ionomer membrane are shown in Figure 6 and demonstrate that the results of the fitting are within the range of experimental data. As can be seen in Figure 6a, the fitted water diffusion coefficient is lower than the majority of the published experimental values. As discussed above, most of the measurements in the literature are ex-situ, with fixed gradients in water concentration across the membrane. The values obtained with the fitting procedure are based on the membrane characteristics in an operating cell: the membrane is sandwiched between two porous layers, under compression, and a temperature gradient exists between anode and cathode interfaces. It has been shown by Kim and Mench [56] that the temperature gradient can influence the water flux in the membrane. Net water flux to the anode can be reduced by thermo-osmosis. This effect is present in an operating cell but not in the model, and the
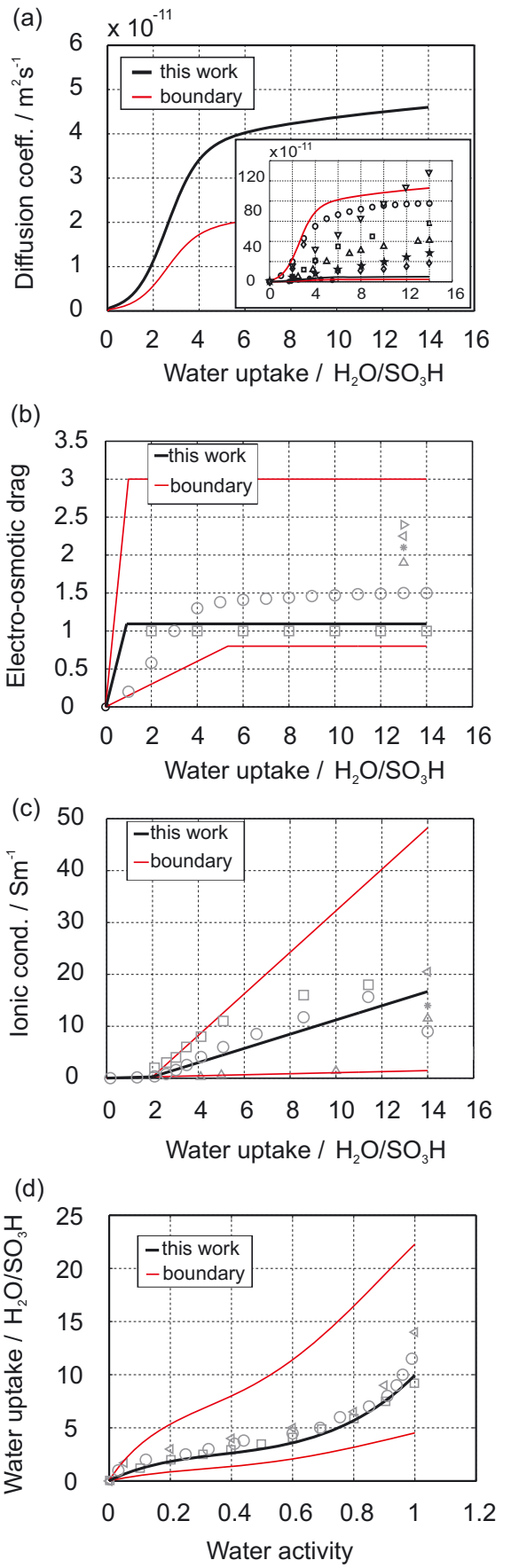

Fig. 6. (Color online) Membrane properties calculated from the fitting procedure: (a) water diffusion coefficient, inset showing the entire seeking field, (b) electro-osmotic drag coefficient, (c) ionic conductivity and (d) water uptake isotherm. Symbols related to Figure 4.

optimization procedure may fit the diffusion coefficient to a lower value.

For the other three fitted parameters, values within the range of the experimental data have been found. The electro-osmotic drag coefficient is in a good agreement with the value determined by Zawodzinski et al. [42] and Jinnouchi et al. [57]. 
The ionic conductivity matches the best values measured by Yang et al. [14] using ac impedance technique and the value at $\lambda=14$ is in the range predicted by Verbrugge et al. [11] between 60 and $77^{\circ} \mathrm{C}$.

Finally, the water isotherm uptake is in very good agreement with the curve measured by Hinatsu et al. [49] at $80^{\circ} \mathrm{C}$ by a gravimetric method.

The GDL porosity was measured in-house by mercury intrusion porosimetry and a value of 0.62 was determined for the uncompressed sample. The fitting procedure determined a value of 0.55 at the anode side and of 0.36 at the cathode side. The lower values are justified by the clamping pressure applied. Moreover, the model does not calculate the local liquid water content in the porous layers, which lowers the effective porosity of the material. At the cathode side the higher liquid water saturation, due to the water generation in the catalyst layer and to the water drag from the anode side, causes a lower effective porosity compared to the anode side. As expected, high tortuosities are found, $\tau_{\text {an }}=8.00$ and $\tau_{\text {cat }}=7.34$. They are in fact the average values between the different paths from the channels to the active layers in the compressed porous domains, while in the ex-situ measurements only the through-plane value is measured.

The results from the fitting procedure show that important material parameters, difficult to obtain in direct experiments, can be extracted from experimental current distribution data with a multiparameter optimization procedure. For most of the parameters, a correspondence with published values is found. The water diffusion coefficient is less accurately described in the literature. In fact, it is a strength of the fitting procedure that effective parameter values are obtained in a running cell.

In order to determine if the fitting procedure converged to the global minimum of the solution field, a sensitivity analysis is performed. Each parameter is changed by $\pm 5 / 10 / 20 / 30 / 40 / 50 \%$, and the RMS deviation between the simulated and the experimental data is calculated. The results are shown in Figure 7. There is a clear minimum for all parameters except for the electro-osmotic drag slope coefficient, B, meaning that the drag coefficient drops to zero for membrane water contents lower that those calculated by the model. This membrane property is then constant over a wide range of operating conditions.

The sensitivity analysis further shows that the fitting procedures converges towards the global minimum in the seeking field. The strongest sensitivity is found for the electro-osmotic drag and the water uptake isotherm. Regarding the porous layers, the model is more sensitive on porosity and tortuosity at the cathode side.

Finally, in Figure 8, the experimental and the calculated current density distributions are shown for the four different operating conditions. Despite the co-/counterflow modes, leading to very different water distributions in the membrane, after the fitting procedure the model is able to predict the performance of the linear cell over a broad range of operating conditions with good agreement. For example, at the cell inlet (0 in Fig. 8) the membrane water content at the membrane-anode GDL in-
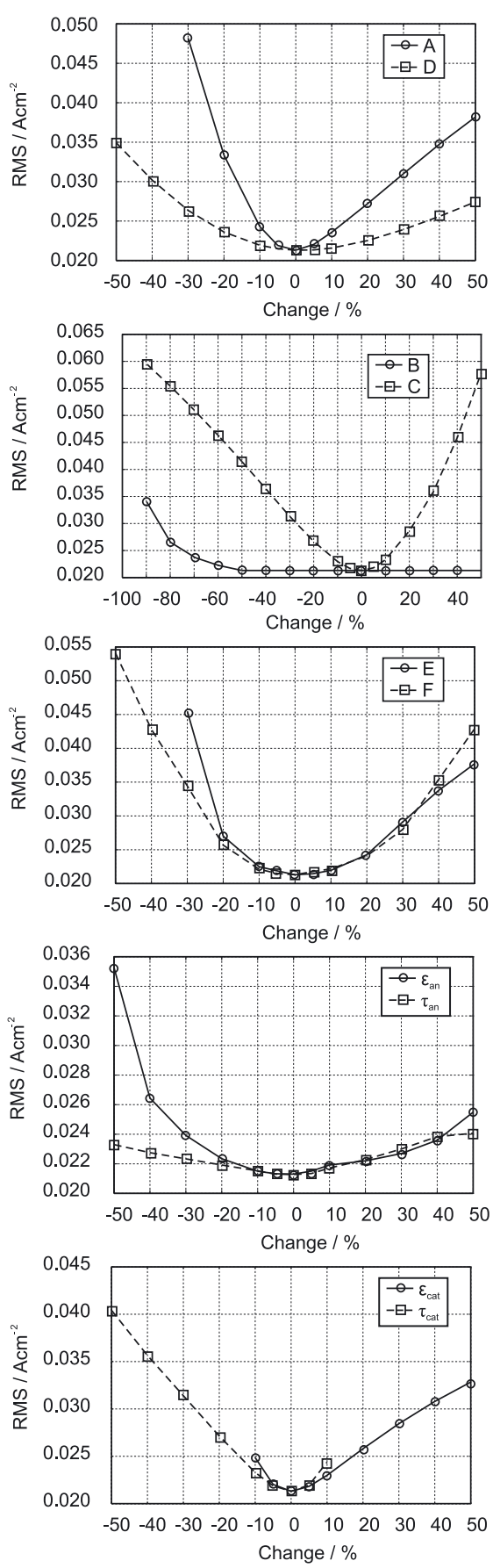

Fig. 7. RMS difference between experimental (symbols) and simulated (lines) data. Each fitted parameter is changed by $\pm 5 / 10 / 20 / 30 / 40 / 50 \%$, except for electro-osmotic drag coefficient, investigated till $-90 \%$. Missing data are due to the nonconvergence of the simulation.

terface ranges from 1 at operating condition "a" to 7.7 at operating condition "c".

\section{Conclusions}

A fitting procedure able to simultaneously extract transport material properties from experimental data measured 
M. Zaglio et al.: PEFC parameter extraction using an evolutionary optimization algorithm

(a)

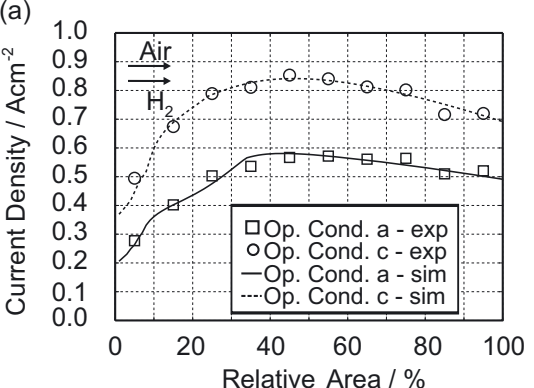

(b)

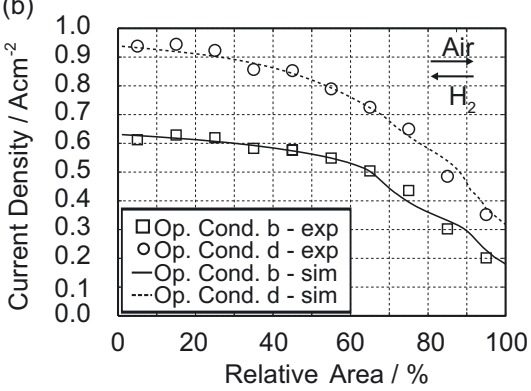

Fig. 8. Comparison between experimental and calculated data after fitting procedure: (a) coflow and (b) counterflow.

in a running cell of technical size has been developed. The procedure, based on an evolutionary algorithm, converges to the set of parameters that minimizes the difference between experimental and simulated current density distributions under significantly different operating conditions. For the ionic conductivity, electro-osmotic drag coefficient, and water isotherm uptake a correspondence with data available in the literature is found, while the water diffusion coefficient in the membrane resulting from the optimization is appreciably lower than the majority of published measurements. This can be due to the inherently different conditions between ex-situ measurements and the operating fuel cell, where the membrane is compressed between the porous layers, and a temperature gradient applies between anode and cathode. The extracted GDL porosities are lower than the measured value, due to compression and liquid saturation. The difference between anode and cathode can be due to the higher saturation in the latter, which is not taken into account by the model. Likewise, the tortuosities are higher than values usually reported in the literature, due to the $1 \mathrm{D}$ approach of the model in the MEA, and therefore to the longer path under the ribs.

A sensitivity analysis confirmed that the solution field presents a well defined global minimum, increasing the reliability on the optimization procedure.

Applying the proper computational tools, determination of material properties, difficult to obtain in direct experiments, can be extracted with good accuracy from experimental current density distributions in technical sized cells. The use of reliable parameters is important for validating and substantiating models and simulations, and can facilitate the further development of the technology by designing cell structures and improving material properties.
We gratefully acknowledge P. Kirchen and the Aerothermochemistry and Combustion Systems Laboratory, ETH Zürich (Switzerland) for providing the evolutionary optimization algorithm.

\section{List of symbols}

$A \quad$ fitting parameter

$a_{w} \quad$ water activity

$B \quad$ fitting parameter

$C \quad$ fitting parameter

$D \quad$ fitting parameter

$E \quad$ fitting parameter

$F \quad$ fitting parameter

$P$ pressure, $[\mathrm{Pa}]$

$R \quad$ universal gas constant, $\left[\mathrm{J} \mathrm{mol}^{-1} \mathrm{~K}^{-1}\right]$

$Y \quad$ RMS deviation, $\left[\mathrm{A} \mathrm{m}^{-2}\right]$

$j$ current density, $\left[\mathrm{A} \mathrm{m}^{-2}\right]$

$j^{o} \quad$ exchange current density, $\left[\mathrm{A} \mathrm{m}^{-2}\right]$

$j^{\text {ref }}$ reference exchange current density, $\left[\mathrm{A} \mathrm{m}^{-2}\right]$

$K$ ionic conductivity, [Sm]

$n_{d} \quad$ nodes along the channel

$T$ temperature, $[\mathrm{K}]$

n electrons involved

\section{greek symbols}

$\alpha \quad$ transfer coefficient

$\epsilon \quad$ porosity

$\lambda$ membrane water content, $\left[\mathrm{H}_{2} \mathrm{O} / \mathrm{SO}_{3} \mathrm{H}\right]$

$\xi \quad$ electroosmotic drag coefficient

$\tau \quad$ tortuosity

\section{subscripts}

$i \quad$ index of summation

vap vapor

sat saturation

\section{superscripts}

exp experimental

sim simulated

ref reference

\section{References}

1. M. Ceraolo, C. Miulli, A. Pozio, J. Power Sources 113, 131 (2003)

2. S.A. Freunberger, M. Santis, I.A. Schneider, A. Wokaun, F.N. Büchi, J. Electrochem. Soc. 153, A396 (2006)

3. T.F. Fuller, J. Newman, J. Electrochem. Soc. 140, 1218 (1993)

4. A. Kulikovsky, Electrochem. Commun. 6, 969 (2004)

5. B.S. Pivovar, Polymer 47, 4194 (2006)

6. E. Springer, T.A. Zawodzinsky, S. Gottesfeld, J. Electrochem. Soc. 138, 2334 (1991)

7. T.A. Zawodzinski Jr., M. Neeman, L.O. Sillerud, S. Gottesfeld, J. Phys. Chem. 95, 6040 (1991)

8. T.V. Nguyen, N. Vanderborgh, J. Membr. Sci. 143, 235 (1998)

9. M.W. Verbrugge, R.F. Hill, J. Electrochem. Soc. 137, 1131 (1990)

10. K.M. Nouel, P.S. Fedkiw, Electrochim. Acta 43, 2381 (1998) 
11. M.W. Verbrugge, E.W. Schneider, R.S. Conell, R. Hill, J. Electrochem. Soc. 139, 3421 (1992)

12. T.A. Zawodzinski, T.E. Springer, J. Davey, R. Jestel, C. Lopez, J. Valerio, S. Gottesfeld, J. Electrochem. Soc. 140, 1981 (1993)

13. D.R. Morris, X. Sun, J. Appl. Polym. Sci. 50, 1445 (1993)

14. C. Yang, S. Srinivasan, A.B. Bocarsly, S. Tulyani, J.B. Benziger, J. Membr. Science 237, 145 (2004)

15. B. Thoben, A. Siebke, J. New Mater. Electrochem. Syst. 7, $13(2004)$

16. J.H. Nam, M. Kaviany, Int. J. Heat Mass Transfer 56, 4595 (2003)

17. S.Y. Lee, K.S. Lee, S. Um, J. Mech. Sci. Technol. 22, 565 (2008)

18. Z.J. Mo, X.J. Zhu, L.Y. Wei, G.Y. Cao, Int. J. Energy Res. 30, 585 (2006)

19. L. Peng, X. Lai, D. Liu, P. Hu, J. Ni, J. Power Sources 178, 223 (2007)

20. W. Na, B. Gou, J. Power Sources 166, 411 (2007)

21. C. Bao, M. Ouyang, B. Yi, J. Power Sources 156, 232 (2006)

22. I. Mohamed, N. Jenkins, J. Power Sources 131, 142 (2004)

23. M. Zaglio, G. Schuler, A. Wokaun, J. Mantzaras, F.N. Büchi, in Proc. of Fundamentals and Developments of Fuel Cells Conf. 2008 (FDFC2008), Nancy, France, 2008, p. 12

24. M. Warth, Ph.D. thesis, ETH, Zürich, Switzerland, 2005

25. A.E. Eiben, J.E. Smith, Introduction to Evolutionary Computing, 1st edn. (Springer, Natural Computing Series, 2003)

26. S.A. Freunberger, I.A. Schneider, P.C. Sui, A. Wokaun, N. Djilali, F.N. Büchi, J. Electrochem. Soc. 153, A909 (2006)

27. F.N. Büchi, A.B. Geiger, R.P. Neto, J. Power Sources 145 , $62(2005)$

28. I.A. Schneider, H. Kuhn, A. Wokaun, G. Scherer, J. Electrochem. Soc. 152, A2092 (2005)

29. S.A. Freunberger, M. Reum, J. Evertz, A. Wokaun, F.N. Büchi, J. Electrochem. Soc. 153, A2158 (2006)

30. M. Reum, S.A. Freunberger, A. Wokaun, F.N. Büchi, J. Electrochem. Soc. 156, B301 (2009)

31. M. Reum, S.A. Freunberger, A. Wokaun, F.N. Büchi, J. Electrochem. Soc. 156, B1225 (2009)

32. D. Bernardi, M. Verbrugge, J. Electrochem. Soc. 139, 2477 (1992)

33. C. Amphlett, R. Baumert, T. Harris, R. Mann, B. Peppley, P. Roberge, J. Electrochem. Soc. 142, 1 (1995)

34. J. Larminie, A. Dicks, Fuel Cell Systems Explained, 2nd edn. (John Wiley \& Sons, 2003)
35. S.C. Yeo, A. Eisenberg, J. Appl. Polym. Sci. 21, 875 (1977)

36. S. Motupally, A.J. Becker, J.W. Weidnera, J. Electrochem. Soc. 147, 3171 (2000)

37. R. Zelsmann, M. Pineri, M. Thomas, M. Escoubes, J. Appl. Polym. Sci. 41, 1673 (1990)

38. T.F. Fuller, Ph.D. thesis, University of California, Berkeley, CA, 1992

39. D. Rivin, C.E. Kendrick, P.W. Gibson, N.S. Schneider, Polymer 42, 623 (2001)

40. H.P.L.H. van Bussel, F.G.H. Koene, R.K.A.M. Mallant, J. Power Sources 71, 218 (1998)

41. T.F. Fuller, J. Newman, J. Electrochem. Soc. 139, 1332 (1992)

42. T.A. Zawodzinski, J. Dawy, J. Valerio, S. Gottesfeld, Electrochim. Acta 40, 297 (1995)

43. M. Ise, K.D. Kreuer, J. Maier, Solid State Ion. 125, 213 (1999)

44. A.V. Anantaraman, C.L. Gardner, J. Elecroanal. Chem. 414, 115 (1996)

45. P.C. Reike, N.E. Vanderborgh, J. Membr. Sci. 32, 313 (1987)

46. T.A. Zawodzinski, C. Derouin, S. Radzinski, R.J. Sherman, V.T. Smith, T.E. Springer, S. Gottesfeld, J. Electrochem. Soc. 140, 1041 (1993)

47. F.N. Büchi, G.G. Scherer, J. Electroanal. Chem. 404, 37 (1993)

48. M. Marechal, J.L. Souquet, J. Guindet, J.Y. Sanchez, Electrochem. Commun. 9, 1039 (2007)

49. J.T. Hinatsu, M. Mizuhata, H. Takenaka, J. Electrochem. Soc. 141, 1493 (1994)

50. N.H. Jalani, P. Choi, R. Datta, J. Membr. Sci. 254, 31 (2005)

51. K.K. Pushpa, D. Nandan, R.M. Iyer, J. Chem. Soc. Faraday Trans. I 84, 2047 (1988)

52. S. Park, B.N. Popov, Fuel 88, 2068 (2009)

53. M.V. Williams, E. Begg, L. Bonville, H.R. Kunz, J.M. Fenton, J. Electrochem. Soc. 151, A1173 (2004)

54. R. Flückiger, S.A. Freunberger, D. Kramer, A. Wokaun, G.G. Scherer, F.N. Büchi, Electrochim. Acta 54, 551 (2008)

55. A. Kulikovsky, J. Electrochem. Soc. 150, A1432 (2003)

56. S. Kim, M. Mench, J. Membr. Sci. 328, 113 (2009)

57. R. Jinnouchi, H. Yamada, Y. Morimoto, in Proc. of 14 th Int. Conf. on the Properties of Water and Steam in Kyoto, Japan, 2004, edited by M. Nakahara, N. Matubayasi, M. Ueno, K. Yasuoka, K. Watanabe (2004), pp. 403-406 